\title{
Comparison of Quantitative POCT Plasma D Dimer Values With Semiautomated Method
}

\author{
R.D.Malathi' ${ }^{1}$ Jyotsna Volturi ${ }^{*}$ and Dawood Suleman ${ }^{3}$ \\ Department of Biochemistry, Gandhi Medical College, Secunderabad. \\ 1, 2 Assistant Professor and ${ }^{3}$ Professor and HOD, MD Biochemistry.
}

Received: 14 Nov 2020 / Accepted: 06 Dec 2020/ Published online: 01 Jan 2021 *Corresponding Author Email: driyotsna.mdgmc@gmail.com

\begin{abstract}
Background and Aim: D Dimer is a soluble fibrin degradation product, the estimation of which is used to identify intravascular thrombosis. Different types of D Dimer assays are available commercially which help to diagnose VTE/PE/DIC. The aim of this study was to compare plasma D Dimer values on POCT and Semi-auto analyser over random samples received in the lab and correlate the performance of both. Method: A prospective observational study conducted in a tertiary care hospital in Telangana. Results: Main outcome measures were the statistical correlation of the two methods. The results showed that there was strong correlation of POCT D-Dimer values with Semi-automated method. The correlation between the two was significant with $r$ value of 0.846 . When subjected to regression analysis, it was not significant indicating that both values were almost comparable and that POCT was not superior to Semi automated method. Conclusion: The POCT device was comparable with existing Semiautoanalyzer and can be used for reliable estimation of D Dimer.
\end{abstract}

\section{Keywords}

D Dimer, POCT, Semi-autoanalyzer, Assay, Comparison.

INTRODUCTION:

D-dimer is a fibrin degradation product (FDP) present in the blood after a blood clot is degraded by fibrinolysis and contains two $D$ fragments of the fibrin protein joined by a cross-link ${ }^{[1]}$.

D-dimer is normally present in low levels in human blood plasma and levels increase when the coagulation system has been activated. D-dimer concentration is determined by a blood test to help diagnose thrombosis/DVT/PE/DIC ${ }^{[1]}$. While a negative result practically rules out thrombosis, a positive result can indicate thrombosis but does not rule out other potential causes. A four-fold increase in the protein is a strong indicator of mortality in those suffering from COVID-19 ${ }^{[2,3]}$.

A D-dimer assay can be helpful provided it is sensitive, reliable, fast and easy to perform. D-dimer assays depend on the binding of a monoclonal antibody to a particular epitope on the D-dimer fragment. The binding of the antibody is then measured quantitatively by one of various laboratory methods ${ }^{[1]}$.

D-dimer standardization is still difficult as it concerns the detection in a complex mixture of fibrin degradation products of different sizes ${ }^{[4-6]}$.There is also lack of a universal calibrator that can be used to standardize D-dimer concentration across assay types ${ }^{[7,8]}$. D-dimer units thus may vary according to the type of calibrator used, i.e. DDU or FEU $[9,10]$. Different labs report different units either as FEU/DDU.

The molecular weight of the fibrinogen molecule is about twice the size of the D-dimer molecule, and therefore $1.0 \mathrm{mcg} / \mathrm{mL} \mathrm{FEU} \mathrm{is} \mathrm{equivalent} \mathrm{to} 0.5$ $\mathrm{mcg} / \mathrm{mL}$ of D Dimer ${ }^{[11]}$. 
Tests based on the ELISA methodology have a high diagnostic sensitivity but the drawbacks are their long assay times, unsuitable for processing single sample when required, and high cost per test. New methods using immunofiltration or by microlatex immunoturbidimetric assays seem to reach the high sensitivity and NPV required and allows fast and quantitative single sample analysis.

Recently, a number of point-of-care D Dimer assays have been developed for acute care settings that utilize a variety of methodologies and had attracted the general physicians because of rapidity of testing. In view of the diversity of D-dimer assays used in central laboratory and point-of-care settings, results with one assay cannot be extrapolated to another [12].

In the present study, we compared the results of quantitative POCT D Dimer (Hotgen) with a Semi automated coagulation analyzer (Erba ECL 412) already present in our lab.

\section{MATERIAL AND METHODS:}

The study was conducted in Biochemistry lab of Gandhi Hospital, Secunderabad. The data was collected from November 2020 to December 2020 involving 47 samples received in the Biochemistry Laboratory for analysis of plasma D Dimer. $3 \mathrm{~mL}$ of venous blood samples was collected in sodium citrate (Blue) vacutainer and was centrifuged at 1500 rpm for 15 minutes to obtain plasma. The plasma was used to estimate D Dimer using Semi automated analyser (ERBA-ECL 412) and simultaneously by POCT instrument (HOTGEN).

\section{Inclusion Criteria:}

1) Samples received in the Biochemistry lab with request for $D$ Dimer test.

2) Samples with correct volume of blood

3) Samples collected in the citrated vacutainers only

\section{Exclusion Criteria:}

1) Insufficient volume of samples

2) Samples collected in incorrect type of vacutainers

3) Clotted samples

4) Improperly labelled samples

Assays:

POCT Hotgen: Upconverting phosphor technology. It employs Sandwich Immunochromatography as an aiding tool.

Reference range according to kitinsert : $<500 \mathrm{ng} / \mathrm{ml}$ FEU

Linearity: upto $5000 \mathrm{ng} / \mathrm{ml}$

ERBA ECL 412: Erba D Dimer is a Immunoturbidimetric assay that utilizes antibody coated latex particles. The antibody has no cross reactivity with fibrinogen ${ }^{[13]}$. This allows for the determination of D Dimer in human plasma.

Reference range according to kitinsert ${ }^{[14]}:<200$ $\mathrm{ng} / \mathrm{ml}$ DDU

Linearity: up to $3200 \mathrm{ng} / \mathrm{ml}$

The approval of the local ethics committee was obtained as was the patient's consent to follow the diagnostic workup adopted in our centre.

Statistical analysis:

The data was presented as Mean, Standard deviation (SD) and number (N). Linear relationships between variables were determined using Pearson correlation test. All statistical analyses were performed using SPSS software, version 20.0.

\section{RESULTS:}

Erba D Dimer values were obtained in $\mathrm{ng} / \mathrm{ml}$ DDU units whereas Hotgen values obtained in $\mathrm{ng} / \mathrm{ml} \mathrm{FEU}$ units. Initially the DDU units were converted to FEU units for comparison between both sets of values. $1 \mathrm{FEU}=2^{*} \mathrm{DDU}{ }^{[12]}$

Now the standard cut-off was, therefore $<500 \mathrm{ng} / \mathrm{ml}$ FEU for both the D-dimer assays.

Table-1 shows the higher mean D Dimer value and SD of Erba when compared to Hotgen mean.

All the D dimer values were grouped into 3 groups. Group 1: values from $50-500 \mathrm{ng} / \mathrm{mL}$ Group 2: $>500-2000 \mathrm{ng} / \mathrm{mL}$ and Group 3: > 2000 $\mathrm{ng} / \mathrm{mL}$. Paired t-test was done on the above data shown in Table- 2 which indicates strong correlation between the pair of values in all the 3 groups with a significant $p$ value of $<0.01$.

Figure-1: shows a comparison of test results between the Hotgen and the Erba D-dimer assays for the entire range of test values measured. Overall, there was positive linear correlation between both the values.

Table-3 shows the relationship between the two sets of values using Pearson Correlation. Accordingly, it was observed that there exists a strong correlation between both the values ( $r=0.846)$ with a significant $p$ value of $<0.01$.

Figure-2: is a Histogram showing normal distribution of mean for the difference between Erba and Hotgen values

It was hypothesized that POCT Hotgen was a better method when compared to Erba, hence the data was subjected to regression analysis in Table-4, but it didn't show significance with a $p$ - value of 0.102 , indicating Hotgen was not superior to Erba.

Figure 3: is a Scatter plot for regression showing no much difference between Erba and Hotgen values 
Table 1- Data

\begin{tabular}{lllr}
\hline & Mean & Std. Deviation & N \\
\hline Method A (ERBA) & 1056.8511 & 1069.20749 & 47 \\
Method B (HOTGEN) & 653.1915 & 709.22605 & 47 \\
\hline
\end{tabular}

Table 2- Paired t-test of grouped data

\begin{tabular}{|c|c|c|c|c|c|}
\hline \multirow{2}{*}{ System } & \multicolumn{5}{|l|}{$50-500$} \\
\hline & Mean & SD & & lue & p-value \\
\hline \multirow{2}{*}{$\begin{array}{l}\text { Erba(ng/mL) } \\
\text { Hotgen(ng/mL) }\end{array}$} & 525.516 & 217.45 & \multirow{2}{*}{\multicolumn{2}{|c|}{-4.83}} & \multirow{2}{*}{$<0.001$} \\
\hline & 262.758 & 108.7274 & & & \\
\hline \multirow{2}{*}{ System } & $>500-2000$ & & & & \\
\hline & Mean & \multicolumn{2}{|c|}{ SD } & t-value & p-value \\
\hline Erba(ng/mL) & 2336.744 & \multicolumn{2}{|c|}{998.92} & \multirow{2}{*}{-4.313} & \multirow{2}{*}{0.0002} \\
\hline Hotgen(ng/mL) & 1168.372 & & & & \\
\hline \multirow{2}{*}{ System } & $>2000$ & & & & \\
\hline & Mean & SD & t-value & \multicolumn{2}{|c|}{ p-value } \\
\hline Erba(ng/mL) & 5369.52 & 1405.541 & \multirow{2}{*}{3.12} & \multirow{2}{*}{\multicolumn{2}{|c|}{0.005}} \\
\hline Hotgen(ng/mL) & 3348.533 & 1488.913 & & & \\
\hline
\end{tabular}

Table 3: Correlations

\begin{tabular}{llll}
\hline & & Method A (ERBA) & Method B (HOTGEN) \\
\hline \multirow{2}{*}{ Method A (ERBA) } & Pearson Correlation & 1 & $.846^{* *}$ \\
& Sig. (2-tailed) & & .000 \\
& $N$ & 47 & 47 \\
Method B (HOTGEN) & Pearson Correlation & $.846^{* *}$ & 1 \\
& Sig. (2-tailed) & .000 & 47 \\
& $\mathrm{~N}$ & 47 & 47 \\
& $* *$ Correlation is significant at the 0.01 level (2-tailed).
\end{tabular}

Table 4: Regression analysis

\begin{tabular}{llllll}
\hline Model & Sum of Squares & df Mean Square & F & Sig. \\
\hline Regression & 1176039.653 & 1 & 1176039.653 & 2.789 & $.102^{b}$ \\
1 Residual & 19399232.823 & 46421722.453 & & \\
$\quad$ Total & 20575272.477 & 47 & & & \\
\hline
\end{tabular}

a. Dependent Variable: $(A-B)$

b. Predictors: (Constant), $(A-B) /$

Figure 1: Linear comparison of two sets of value

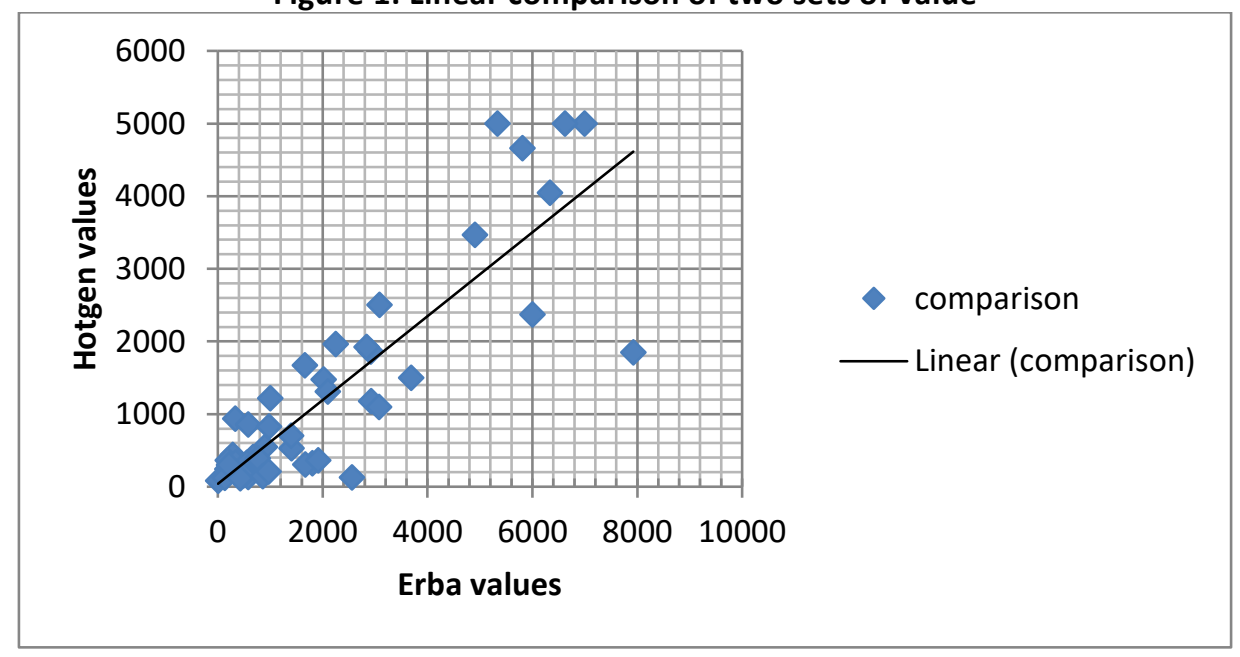


Figure-2: Histogram showing normal distribution.

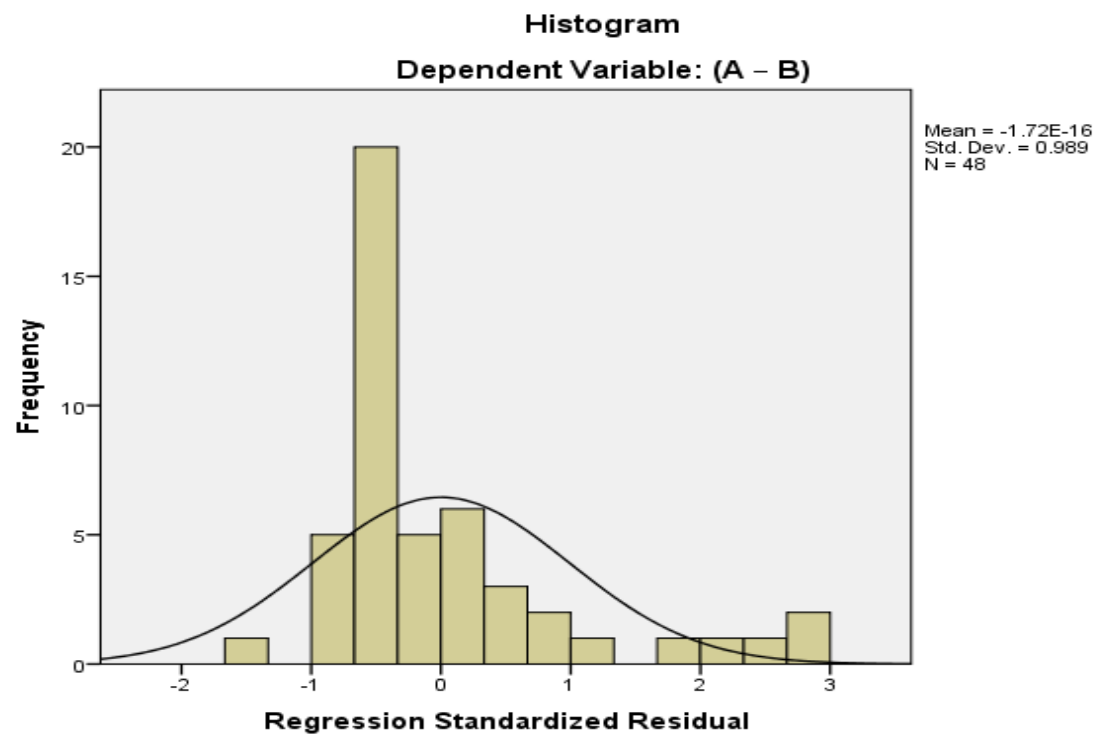

Figure 3: Scatter plot for regression

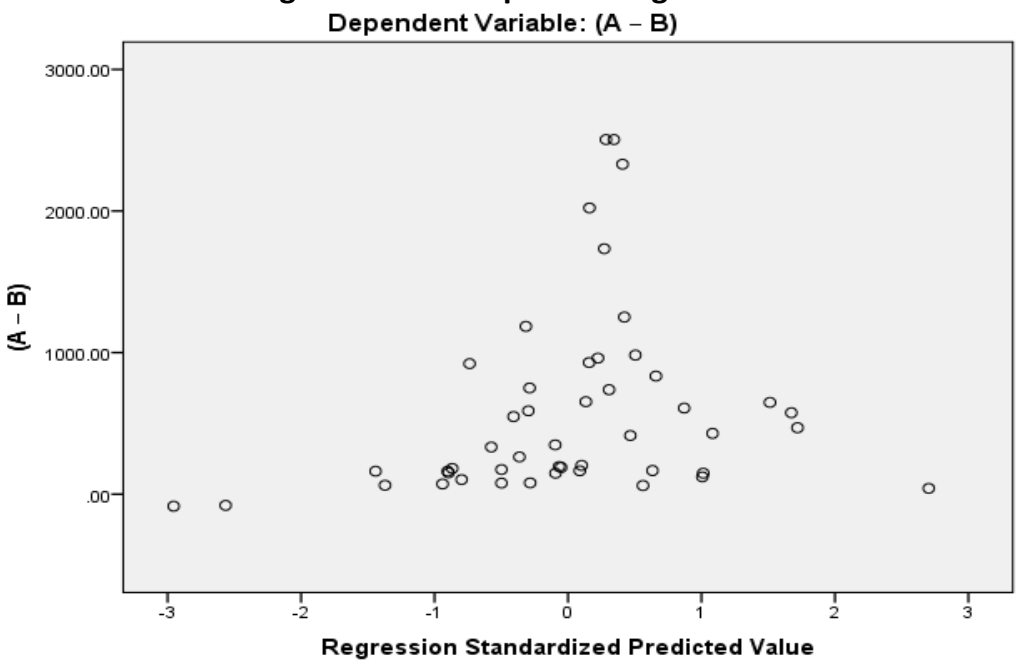

\section{DISCUSSION:}

D-dimers are formed by the breakdown of fibrinogen and fibrin during fibrinolysis. D-dimer analysis is critical for the diagnosis of deep vein thrombosis, pulmonary embolism, and disseminated intravascular coagulation ${ }^{[15]}$.

Our study compared the D Dimer values in two different methods (Immunochromatography-POCT and Turbidimetry- Semiautomated analyzer) with different units (POCT Hotgen-FEU units and ErbaDDU units). Both D-dimer assays were compared with each other in order to determine the analytical range of measurement.

The use of different units (FEU or DDU) is challenging for clinicians, causes confusion and potentially leads to the misclassification or misdiagnosis of patients [16]. Here the Erba D Dimer values were mathematically converted to FEU units.
D Dimer values were segregated into 3 groups and paired t-test done over the data. Significant correlation was found in all the three groups indicating the values of POCT were comparable to that of semiautomated method.

The whole data was then subjected to Pearson correlation. The $r$ value $(0.846)$ indicated a strong correlation between the two sets of values with a significant $p$ value $<0.01$ similar to that of Fukuda T et al study ${ }^{[17,18]}$.

In the present study, $85 \%$ values positive on Erba were also positive on Hotgen which is in concordance with the findings of Perveen et al ${ }^{[19]}$. This could be due to improper dilution, incorrect incubation time or results read after more than 15 minutes etc. The mean difference between the values showed a normal distribution.

According to Lee-Lewandrowski et al study, the POCT D Dimer values were lower when compared to 
automated instrument ${ }^{[20]}$. Similar finding was obtained in the present study also.

The FEU is based on the mass of fibrinogen ( $340 \mathrm{kDa}$ ) and is approximately 1.75 -fold higher than the DDU, which is based on the weight of the D-dimer ${ }^{[21]}$. In this study, it was taken as 2 which could be the reason that the mean Erba values were little on higher side when compared to Hotgen values.

Later the data was subjected to Regression analysis which showed that values produced by Hotgen were comparable with that of Erba and not superior to Erba.

\section{CONCLUSION:}

The POCT- Hotgen D-dimer method was comparable to Erba -Semi automated method for reliable estimation of D-dimer provided the values are reported with caution considering the units of FEU and DDU. It is necessary that we focus on uniformity of reporting units while reporting using different types of assays available in the market.

Acknowledgement: The authors would like to thank the staff of Gandhi Medical College /Hospital. The authors also wish to acknowledge Dr.N.Lavanya, Scientific Officer for providing assistance in statistics.

\section{REFERENCES:}

1. Adam SS, Key NS, Greenberg CS. "D-dimer antigen: current concepts and future prospects". Blood. 2009; 113 (13): 2878-87.

2. James Hamblin. Why Some People Get Sicker Than Others.COVID-19 is proving to be a disease of the immune system. This could, in theory, be controlled. https://www.theatlantic.com/health/archi ve/2020/04/coronavirus-immune-response/61022 8/ April 21, 2020

3. Velavan, Thirumalaisamy P.; Meyer, Christian G. "Mild versus severe COVID-19: laboratory markers". International Journal of Infectious Diseases. doi:10.1016/j.ijid.2020.04.061. Retrieved 25 April 2020.

4. Righini $\mathrm{M}$, Perrier A, De Moerloose $\mathrm{P}$, Bounameaux $\mathrm{H}$. D-Dimer for venous thromboembolism diagnosis: 20 years later. J Thromb Haemost 2008; 6:1059-1071.

5. Gaffney PJ, Edgell T, Creighton-Kempsford L, Wheeler $\mathrm{S}$, Tarelli E. Fibrin degradation product (FnDP) assays: analysis of standardization issues and target antigens in plasma. Br J Haematol 1995; 90:187-194.

6. Nieuwenhuizen W. A reference material for harmonisation of $D$-dimer assays. Fibrinogen Subcommittee of the Scientific and Standardization Committee of the International Society of Thrombosis and Haemostasis. Thromb Haemost 1997; 77:10311033.

7. Longstaff C, Adcock D, Olson JD, et al. Harmonisation of D-dimer - A call for action. Thromb Res. 2016; 137:219-220.
8. Olson JD, Cunningham MT, Higgins RA, Eby CS, Brandt JT. D-dimer: simple test, tough problems. Arch Pathol Lab Med. 2013;137:1030-1038.

9. Reber G, De Moerloose P. Standardization of D-dimer testing. In: Kitchen S, Olson JD, Preston FE, editors. Quality in laboratory hemostasis and thrombosis. 2nd ed. Oxford, UK: John Wiley \& Sons; 2013. p. 136-146.

10. Dempfle CE, Zips S, Ergul $\mathrm{H}$, et al. The fibrin assay comparison trial (FACT): correlation of soluble fibrin assays with D-dimer. Thromb Haemost. 2001;86: 1204-1209.

11. "Clinical Education Center". Quest Diagnostics .Document FAQS.149 Version: 3 effective 07/23/2019 to present. http://education.questdiagnostics.com/fa q/FAQ149

12. L.-A. Linkins,S. Takach Lapner. Review of D-dimer testing: Good, Bad, and Ugly. Int J Lab Hem. 2017;39(Suppl. 1):98-103.

13. Holvoel $P$ et al. (1989) Binding properties of monoclonal antibodies against human fragment $D$ Dimer of cross-linked fibrin to human plasma clots in an in vivo model in rabbits. Thrombosi and Haemostatsis, 61:307-313.

14. Lindahl $T$ et al. Clinical evaluation of diagnostic strategy for deep venous thrombosis with exclusion by low plasma levels of fibrin degradation product $D$ dimer. Scand. J. Clin, Lab, Invest, 1998;58:307-316

15. Roger S. Riley, Andrea R. Gilbert, Justin B. Dalton, Sheela Pai, and Richard A. McPherson. Widely Used Types and Clinical Applications of D-Dimer Assay. Laboratory Medicine. 2016;47:2:90-102

16. Thachil J, Lippi G, Favaloro EJ. D-dimer testing: laboratory aspects and current issues. Methods Mol Biol. 2017; 1646:91-104.

17. Fukuda T, Kasai H, Kusano T, Shimazu C, Kawasugi K, Miyazawa $Y$. A rapid and quantitative D-Dimer assay in whole blood and plasma on the point-of-care PATHFAST analyzer. Thromb Res. 2007;120(5):695701. doi:10.1016/j.thromres.2006.12.021

18. Waser G, Kathriner S, Wuillemin WA. Performance of the automated and rapid STA Liatest D-dimer on the STA-R analyzer. Thromb Res. 2005;116(2):165-170. doi:10.1016/j.thromres.2004.12.003

19. Perveen S, Unwin D, Shetty AL. Point of care D-dimer testing in the emergency department: a bioequivalence study. Ann Lab Med. 2013;33(1):3438. doi:10.3343/alm.2013.33.1.34

20. Elizabeth Lee-Lewandrowski, John Nichols, Elizabeth Van Cott, Ricky Grisson, Abner Louissaint, Theodore Benzer, Kent Lewandrowski, Implementation of a Rapid Whole Blood D-Dimer Test in the Emergency Department of an Urban Academic Medical Center: Impact on ED Length of Stay and Ancillary Test Utilization, American Journal of Clinical Pathology, 2009;132(3):326-331

21. Olson JD. D-dimer: An overview of hemostasis and fibrinolysis, assays, and clinical applications. Adv Clin Chem. 2015; 69:1-46. 